\title{
A NEW METHOD TO IMPROVE THE TOTAL EFFICIENCY OF PARALLEL CONVERTERS
}

\author{
Fabrício Hoff Dupont ${ }^{1}$, Jordi Zaragoza ${ }^{2}$, Cassiano Rech $^{1}$, José Renes Pinheiro ${ }^{1}$ \\ ${ }^{1}$ Power Electronics and Control Research Group (GEPOC) — Federal University of Santa Maria (UFSM) \\ Av. Roraima, 1000, 97105-900, Santa Maria, Rio Grande do Sul, Brazil \\ ${ }^{2}$ Terrassa Industrial Electronics Group (TIEG) — Technical University of Catalonia (UPC) \\ C. Colom, 1, 08222, Terrassa, Barcelona, Spain \\ Contact: fhd@ieee.org
}

\begin{abstract}
This paper proposes a new methodology for efficiency optimization in systems composed by parallel converters. The proposed methodology takes into account the individual efficiency curves and determines the optimum operating point for each converter such that the maximum efficiency of the arrangement is achieved for the entire load range. Due to the nonlinearity of the problem and the complexity of the solution hyperplane, the optimization process is divided into stages of global optimization, local optimization and ambiguity resolution. This latter verifies the existence of multiple global minima and selects the most appropriate in function of previous power distributions. Case studies demonstrates the validity of the proposed methodology for different systems configurations.
\end{abstract}

Keywords-Parallel converters, supervisory control, efficiency maximization, photovoltaic systems

\section{INTRODUCTION}

The growing applications of renewable power sources and the increasing development of distributed power generation are providing great incentives to the power electronics market. This development has being motivated mainly by environmental concerns, with the need to use cleaner power sources. Among renewable sources, the most employed are wind and photovoltaic, and due to their high installation costs, it is of fundamental importance that the maximum generated energy be delivered to the loads or to the grid. This helps to reduce the return time over the investments and allows a better use of the generation installed capacity.

One of the main characteristics of wind and solar energy is the variability of the generated energy due to environmental aspects. Therefore, converters in charge of the interface between generation and consumption are constantly submitted to variations in its operating points. For the case of solar energy, Dupont et al. shows that for the Brazilian territory the large share of the processed power by the photovoltaic modules is found between $20 \%$ and $60 \%$ of the peak installed power [1]. However, even converter topologies or soft switching techniques developed to reduce losses present low efficiency in light load situations as, for example, the approaches presented by [2], [3], [4].

One way to overcome this problem is the undersizing of the power converts, aiming to shift the highest eficiency region near to the power range that the photovoltaic system will operate most of its time [5]. In this manner, although limiting the power handling capacity, one can improve the overall system efficiency such that in long-term this limitation is compensated.

Another approach to improve the overall system efficiency is the use of parallel connected converters [6]. Arrangements like these allows one to use modular converters, with smaller power ratings and employing semiconductors with reduced losses. Besides it enables the use of a minimum number of operating converters to fulfill the load demand. However, a control and a supervision strategy that allows the right power distribution among converters arises as a problem. This power sharing should also enable the system to achieve the maximum efficiency for all load values inside the operational limits of the converters.

Efficiency improvement in systems of parallel converts has been a research theme for some recently works. Zumel et al. employ an interleaved buck converter and dynamically varies the number of active phases as a function of load demand, aiming to achieve loss reduction in light load operation [7]. Abu-Qahouq et al. investigate the effects that several power distributions among the phases of an interleaved buck converter have on the efficiency of the converter. From this analysis the authors propose an algorithm that promotes the power distribution among the phases after a given number of switching events with the objective to find the distribution that provides a better efficiency [8]. However, for systems with larger number of phases, the disturbance algorithm can become too complex. A passive current sharing method is proposed by Kelly and is based on the first-order digital sinc filter [9]. This method aims to achieve the current balance in a cycle-by-cycle manner, improving the transient response. On the other hand, the author observes that the employed filtering could be a problem in systems of three or more converters.

The main objective of this paper is to propose a new approach to power sharing strategies by means of the design of an efficiency optimization methodology for systems composed by parallel converters. For any number of converters, accompanied by their efficiency curve parameters, the methodology must find the optimal power distribution among converters in order to achieve the maximum system efficiency for all its operating points. The problem formulation and the basic stages of the proposed methodology will be presented in the following sections. Finally, case studies that show the employment of the proposed methodology for different systems configurations are presented. 


\section{System EFFICIENCY AND PROBlEM Formulation}

The efficiency $\eta$ of static converters is defined by the relationship between the output power delivered to the loads $\left(p_{\text {out }}\right)$ and the input power $\left(p_{\text {in }}\right)$ provided by the source, i.e.

$$
\eta=\frac{p_{\text {out }}}{p_{\text {in }}} .
$$

Losses in the conversion process arises from several ways, either by conduction or switching actions of semiconductors, ancillary systems such as drive circuits, protection or signaling, among other phenomena [10], [11].

The total losses of the converter varies depending on its operating point, as well as in terms of their technological and constructive characteristics. For light loads, losses in drive circuits tend to be more significant and also approximately constant. On the other hand, increasing power levels turn the switching and conduction losses more significant.

Efficiency of power converters are commonly presented as graphics for all the load range that the converter is able to handle. As seen in the literature the efficiency curves of converters can be approximated, except in particular cases, by the second order function

$$
\eta\left(p_{\text {in }}\right)=\frac{\alpha_{1} p_{\text {in }}+\alpha_{0}}{p_{\text {in }}^{2}+\beta_{1} p_{\text {in }}+\beta_{0}}
$$

being $\alpha_{1}, \alpha_{0}, \beta_{1}$ and $\beta_{0}$ coefficients that can be obtained by curve fitting algorithms over experimental or simulation results and $p_{\text {in }}$ the input power of the converter.

Thus, in a system composed by $n_{c}$ parallel converters, the global efficiency could be determined by the same relationship between input and output power as

$$
\begin{aligned}
\eta\left(p_{\text {in }, 1}, \ldots, p_{\text {in }, n_{\mathrm{c}}}\right) & =\frac{p_{\text {out }, 1}+\ldots+p_{\text {out }, n_{\mathrm{c}}}}{p_{\text {in }, 1}+\ldots+p_{\text {in }, n_{\mathrm{c}}}} \\
& =\frac{\sum_{m=1}^{n_{\mathrm{c}}} p_{\text {out }, m}}{\sum_{m=1}^{n_{\mathrm{c}}} p_{\text {in }, m}} .
\end{aligned}
$$

Applying (2) in (3), and knowing that $p_{\text {out }}=p_{\text {in }} \eta\left(p_{\text {in }}\right)$, one can evaluate the efficiency of a set of converters. Inspecting (3) it is reasonable to suppose that, for the same input power, the resulting efficiency will be different for each power distribution among converters. This leads to the need of a methodology that is able to establish specific power requirements for each converter in order to ensure that the global efficiency is maximized for each operating point.

Thus, substituting (2) in (3) and rewriting as an optimization problem, one has

$$
\begin{aligned}
& \eta\left(p_{1}, \ldots, p_{\text {in }, n_{\mathrm{c}}}\right)_{\text {máx }}= \\
& \min _{p_{\text {in }}}\left(-\frac{\sum_{m=1}^{n_{\mathrm{c}}} \frac{p_{\text {in }, m}\left(\alpha_{1, m} p_{\text {in }, m}+\alpha_{0, m}\right)}{p_{\text {in }, m}^{2}+\beta_{1, m} p_{\text {in }, m}+\beta_{0, m}}}{\sum_{m=1}^{n_{\mathrm{c}}} p_{\text {in }, m}}\right)
\end{aligned}
$$

whose solution enable the obtention of an optimal set of $p_{\text {in, } m}$ values, ensuring the maximum system efficiency for all possible operating points.
However, the problem (4) is subjected to constraints. One of them establishes that the sum of all powers of the converters must be equal to the power under optimization $\left(p_{\text {otim }}\right)$, i.e., $p_{\text {otim }}=p_{\text {in }, 1}+\ldots+p_{\text {in }, n_{c}}$. This way, one has a linear equality to be respected, which is defined by

$$
\left[\begin{array}{cccc}
1 & 1 & \cdots & 1 \\
0 & 0 & \cdots & 0 \\
\vdots & \vdots & \ddots & \vdots \\
0 & 0 & \cdots & 0
\end{array}\right]\left[\begin{array}{c}
p_{\text {in }, 1} \\
p_{\text {in }, 2} \\
\vdots \\
p_{\text {in }, n_{c}}
\end{array}\right]=\left[\begin{array}{c}
p_{\text {otim }} \\
0 \\
\vdots \\
0
\end{array}\right] .
$$

Besides, another constraint imposed to the solution of (4) states that the input power of each converter $\left(p_{\text {in, } n_{c}}\right)$ must be less than or equal to its maximum value $\left(p_{\text {in, } n_{c}, \text { máx }}\right)$. Thus, one has the linear inequality given by

$$
\left[\begin{array}{cccc}
1 & 0 & \cdots & 0 \\
0 & 1 & \cdots & 0 \\
\vdots & \vdots & \ddots & \vdots \\
0 & 0 & \cdots & 1
\end{array}\right]\left[\begin{array}{c}
p_{\text {in }, 1} \\
p_{\text {in }, 2} \\
\vdots \\
p_{\text {in }, n_{c}}
\end{array}\right] \leq\left[\begin{array}{c}
p_{\text {in, }, \text { máx }} \\
p_{\text {in }, 2, \text { máx }} \\
\vdots \\
p_{\text {in }, n_{c}, \text { máx }}
\end{array}\right] .
$$

Finally, the maximum power that each converter is able to handle must be respected. Then, the search for solutions must be bounded by the interval

$$
\left[\begin{array}{c}
0 \\
0 \\
\vdots \\
0
\end{array}\right] \leq\left[\begin{array}{c}
p_{\text {in }, 1} \\
p_{\text {in }, 2} \\
\vdots \\
p_{\text {in }, n_{c}}
\end{array}\right] \leq\left[\begin{array}{c}
p_{\text {in }, 1, \text { máx }} \\
p_{\text {in }, 2, \text { máx }} \\
\vdots \\
p_{\text {in }, n_{c}, \text { máx }}
\end{array}\right]
$$

As observed below, the optimized function (4) is nonlinear with restrictions. Moreover, due to its nature it can feature multiple local minima, or even multiple global minima, which is generally verified in systems composed by equal converters. To solve this optimization problem, this paper proposes a method to overcome these restrictions and determine the optimal power distribution for each converter. Thus the system will be able to operate at optimal efficiency throughout its entire load range.

\section{Proposed Methodology}

To achieve the goals of the presented efficiency optimization problem, the methodology must be able to perform a search in all the solution hyperplane (global optimization capability) and at the same time provide a good precision in defining the optimal solution (local optimization capability). Besides, occurring multiple global minima, some strategy must arbitrate which is the most appropriate solution for each situation (ambiguity resolution strategy). These three characteristics define the basis for the development of the proposed methodology, whose flowchart is depicted by Fig. 1.

\section{A. Stage 1: initialization}

The first criterion to be defined for the application of the proposed methodology is the specification of its execution mode. One can choose to optimize a single point or even the entire load range of the system. The difference between both modes lies in the fact that for a single solution the Stage 4, for ambiguity resolution, is not executed. In the 


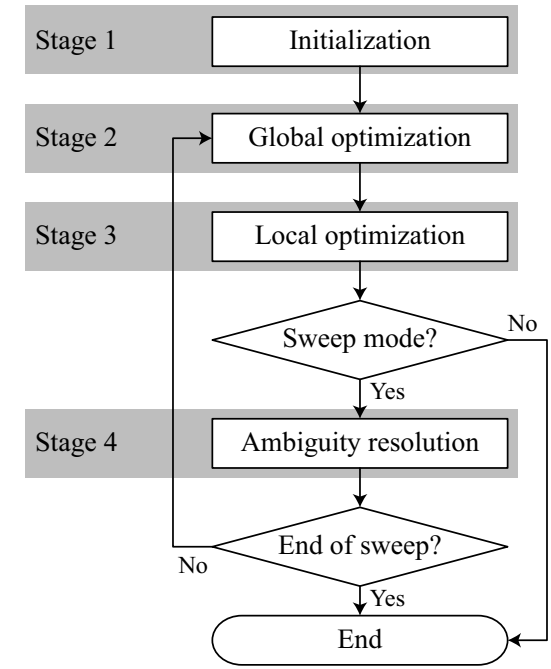

Figure 1: General flowchart for the proposed methodology for efficiency optimization.

initialization stage the system characteristics, as the number of converters, its maximum power and the coefficients $\alpha_{1}$, $\alpha_{0}, \beta_{1}$ and $\beta_{0}$ of the efficiency curves are defined. Based on both the optimization problem (4) and the constraint matrices $(5,6$, and 7$)$ an optimization algorithm is built.

\section{B. Stage 2: global optimization}

The second stage in the proposed methodology refers to a procedure of global optimization with the objective to find a good estimate of the maximum efficiency point according to the constraints imposed to the optimization problem and the possibility of local minima in the solution hyperplane. These characteristics could present a serious problem for most part of numerical optimization algorithms.

Methods like Newton-Raphson, secant, bissecant, among others [12], require an initial guess of the solution, which may not always be possible or easy to obtain. Besides, since they are based in gradients, jacobians, and other derivative terms, these algorithms are sensitive to the inicial guess. If the estimate is not adequately chosen, the algorithm could erroneously converge to a local minimum. For this reason, it is vital that the chosen strategy be able to perform the search for several sections of the solution hyperplane, allowing a general evaluation of the possible solutions before converging to a global minimum point.

The genetic algorithm (GA) is a search and optimization technique based on the natural selection principle, and is one of the techniques for evolutive computing that is most used. The GA are iterative and stochastic algorithms whose goal is to minimize an objective function by means of evolutionary adaptation of a population composed of possible solutions. Among its features it is worth to mentioning that the GA is a robust method that do not need information about the objective function derivatives and could optimize variables in objective functions extremely complex or non-differentiable. Moreover, it can search different sections of the solution hyperplane simultaneously [13], [14].
Populations are sets composed of $n_{\mathrm{i}}$ chromosomes, or individuals, that represents all the variables to be optimized in the objective function (in this case the power processed by each converter). Each chromosome is composed by a total of $n_{\mathrm{g}}$ genes which represents the variables of the problem. The GA is initialized with a random population of chromosomes, and by means of operations similar to those that occur in nature (as generation of offspring and mutation) evolve under specific rules to optimize the objective function.

The first step of the algorithm is the creation of the initial population, which is made from random values that satisfies all the constraints (5)-(7). After, the objective function (4) is evaluated for each chromosome and the results are sorted according to its fitness. A rate $x_{\text {sel }}$ with the worst results are discarded, and the remaining chromosomes are used to generate offspring.

In this paper the choice of parents is carried out employing the roulette wheel method, which assigns a selection probability for each chromosome surviving to natural selection [13]. Offspring are then generated in pairs by means of the scattered crossover method, which creates a random binary vector whose length is equal to the number of genes. For each 1 in this vector, the corresponding gene is copied from the first parent. When 0 , the offspring inherits genes from the second parent. The second offspring is generated in a complementary way. This process is repeated until the population size $n_{\mathrm{i}}$ is reached. In the sequence, the GA applies random mutations in a given number of genes of the population. This mutations have the objective to insert new information and contribute with population diversity. The objective function is then evaluated again for the new population and the process is restarted.

The evolution process follows indefinitely, trying to get a better adjusted population in each new generation. Thus, one need to introduce a stopping criterion for the optimization process. Some of the common criteria are the limitation to a maximum number of generations, or when the best fitness does not evolve after a certain number of generations. Besides, one can employ more sophisticated stopping criteria which identify a possible premature convergence [15].

Due to its stochastic nature, it is possible that the GA may not present an exact solution, or with the required precision, of the global minimum value of the objective function. Moreover, these algorithms are not the best solution to solve all and any problem. Traditional optimization methods have been extensively developed to quickly determine the solution of well defined convex functions. On the other hand, they feature the problem of the initial guess. From a tradeoff between pros and cons of each technique, then arises the alternative to combine the potential of the GA in determining the global minimum in complex hyperplane with the speed of a local optimizer. This is carried out in the next stage of the proposed methodology.

\section{Stage 3: local optimization}

As soon as the GA find a good estimate of the global minimum the local optimization stage is started, where the best chromosome obtained by GA is employed as initial guess for a local optimizer. In turn, this algorithm is 
used to accurately determine the power distribution between converters that provide the optimal system efficiency for a given operation point.

In constrained optimization processes such as this, most approaches transform the main problem in a simpler subproblem, without constraints, and employ a penalty function for results near or outside the limits of constraints. Nowadays this approach is considered inefficient and has been substituted by methods that solve the Karush-KuhnTucker (KKT) equations, which are necessary conditions to the optimization of constrained problems [16]. The KKT equations can be expressed as

$$
\begin{array}{rlrl}
\nabla f\left(\mathbf{x}^{*}\right)+\sum_{i=1}^{m} \lambda_{i} \nabla G_{i}\left(\mathbf{x}^{*}\right) & =0 & & \\
\lambda_{i} G_{i}\left(\mathbf{x}^{*}\right) & =0 & & i=1, \ldots, m_{e} \\
\lambda_{i} & \geq 0 & i=m_{e}, \ldots, m
\end{array}
$$

being $\mathrm{x}$ a vector with length $n$ that corresponds to the number of parameters to be optimized, $f(\mathbf{x})$ is an objective function that returns a scalar value, $G(\mathbf{x})$ is a function that returns a vector with length $m$ containing the equality and inequality values of the constraints evaluated in $\mathbf{x}$, and $\lambda_{i}$ is the Lagrangian multiplier needed to balance the deviations in magnitude of the objective function and the gradients of constraints.

The solution of KKT equations form the basis of many algorithms for nonlinear programming, among them the Sequential Quadratic Programming (SQP), so called once a quadratic programming (QP) subproblem is solved in each major iteration.

The SQP performs an inline search using a figure of merit similar to the proposed in [17], [18]. Also, the SQP approximate the Newton method for constrained optimization as is done for unconstrained problems. At each iteration an approximation of the Hessian is performed, which is given by

$$
\nabla_{x x}^{2} L(\mathbf{x}, \lambda)=\nabla^{2} f(\mathbf{x})+\sum \lambda_{i} \nabla^{2} G_{i}(\mathbf{x})
$$

that is the second derivatives of the Lagrangian

$$
L(\mathbf{x}, \lambda)=f(\mathbf{x})+\sum \lambda_{g, i} G_{i}(\mathbf{x})
$$

employing a quasi Newton updating method. The optimization procedure based on the sequential quadratic programming employed in this paper is described in detail in [19].

When the convergence of the local optimizer is detected, the algorithm is stopped and one has the optimal power distribution among the converters such that the maximum system efficiency is achieved for that operation point.

\section{Stage 4: ambiguity resolution}

In systems of two or more parallel converters with identical specifications one can observe that the objective function (4) may feature multiple global minima. This means that different power distributions can be attributed to the converters, but in these points the same efficiency will be reached, and it will be maximum.
Table I: Coefficients for the efficiency curves employed in the case studies.

\begin{tabular}{c|cccc}
\hline Curve & $\alpha_{1}$ & $\alpha_{0}$ & \multicolumn{1}{c}{$\beta_{1}$} & $\beta_{0}$ \\
\hline$\eta_{A}$ & 7.317 & -0.081 & 5.85 & 0.77 \\
$\eta_{B}$ & 5.072 & -0.037 & 4.4 & 0.18 \\
$\eta_{C}$ & 8.249 & -0.113 & 5.45 & 2.15 \\
\hline
\end{tabular}

Since the GA is a stochastic process, one can not guarantee its convergence to the same region of the global minimum at each new iteration (in case of multiple global minima). Thus, there is no way to ensure that a given power distribution sequence will be employed in a similar manner for the next point when the methodology is running in sweep mode. For this reason, an ambiguity resolution strategy must be employed so that, in case of multiple global minima, the methodology can chose the most appropriate solution against qualitative variables that are not included in the objective function.

To overcome this problem, the ambiguity resolution strategy sorts the results of the power distributions obtained in the previous and current iterations. If the order between the two iterations remains the same, the power distribution obtained by the local optimization algorithm is retained and the next iteration is started. On the other hand, power distributions are sorted according to the previous iteration (i.e. the converter that processed more power remains with the largest share of energy produced and so on), and the resulting efficiency is compared with the efficiency obtained in the current iteration. If both are the same (occurrence of multiple global minima), the order of the previous iteration is maintained. Otherwise, the power distribution obtained by the local optimizer in the current iteration is retained.

By means of this strategy, the problem of sudden power redistributions without achieving improvements in the system efficiency is avoided. Thus, power distribution trajectories are kept as smooth as possible for the entire load range that the system is able to operate.

\section{CAse Studies}

The case studies presented in this section aim to demonstrate and validate the efficiency optimization proposed in this paper. For this, three efficiency curves will be employed, whose coefficients are given in Table I. Notice that the evaluated cases do not particularize any specific topology, semiconductor technology, or assembly of a given converter.

\section{A. Case 1: two different converters}

The first analyzed case involves two different converters with different nominal powers and different efficiency curves. Converter 1 has a nominal power of $0.6 \mathrm{pu}$ and its efficiency curve is described by $\eta_{A}$, while the efficiency curve of Converter 2 is given by $\eta_{B}$ and its maximum power is $0.4 \mathrm{pu}$. For this system, the efficiency surface for all the possible power distributions is depicted by Fig. 2 .

Using the system characteristics and applying them in the proposed methodology, the power distribution curves 


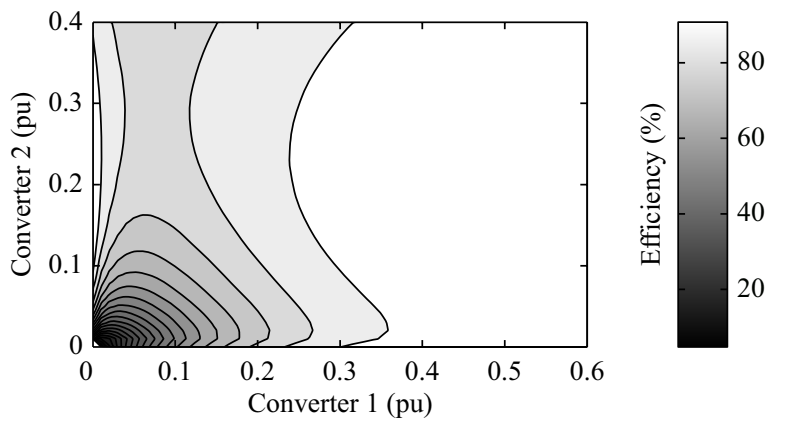

Figure 2: Surface of possible obtained efficiencies for different power distribution between converters 1 and 2 .

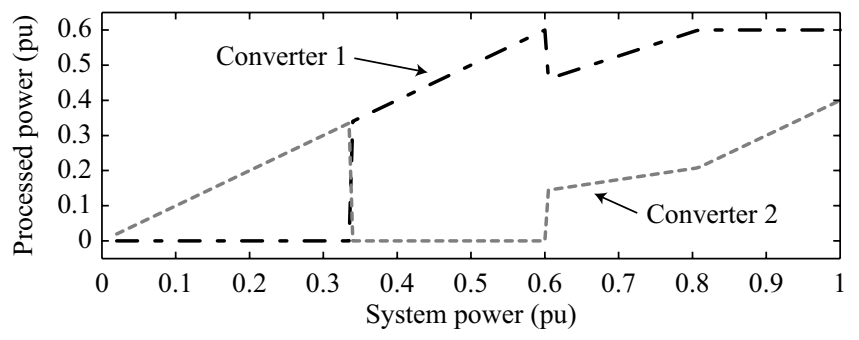

Figure 3: Optimal power distributions obtained by the proposed methodology for Case 1 .

shown by Fig. 3 are obtained. To validate these results, Fig. 4 shows the efficiency values that can be achieved to four random operating points. In this case, $0.28 \mathrm{pu}$, $0.5 \mathrm{pu}, 0.57 \mathrm{pu}$ and $0.78 \mathrm{pu}$. Being $p_{1}$ and $p_{2}$ the power of converters 1 and 2, respectively, the horizontal axis represents the power difference $p_{2}-p_{1}$ between converters. Thus, when $p_{2}-p_{1}=0$ the converters process the same power. The resulting efficiency for each power distribution is given in the vertical axis. Comparing the results of Fig. 4 with the curves of Fig. 3 one can notice that the proposed methodology is able to find and guarantee that the system is capable to operate with maximum efficiency for every operation point.

\section{B. Case 2: three equal converters}

The second case investigates the convergence of the algorithm for a system composed by three converters with equal power ratings and equal efficiency curves. In this case, Converters 1, 2 and 3 has its efficiency curves described by $\eta_{C}$ and its maximum power is $1 / 3 \mathrm{pu}$. The symmetry of this configurations aims to also validate the ambiguity resolution strategy, Stage 4 of the proposed methodology.

The results obtained after running the proposed methodology for this case are depicted by Fig. 5. This figure shows the optimal power distribution among the converters in order to ensure the maximum efficiency of the system for the whole power range.

Fig. 6 validates the methodology to other four operating points. Again, the horizontal axis represents the power difference between converters 1 and 2 , while the vertical axis represent the power handled by Converter 3 . The surface in turn represents the possible efficiency values that could be obtained from the different power distributions. In this case, the symmetry of the problem can be clearly seen, as well as the need of the ambiguity resolution stage to

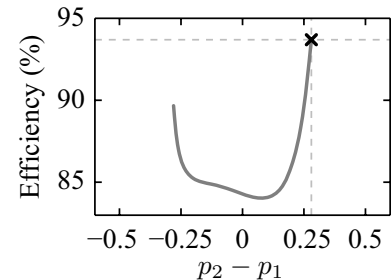

(a)

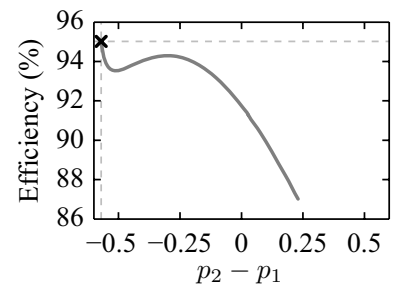

(c)

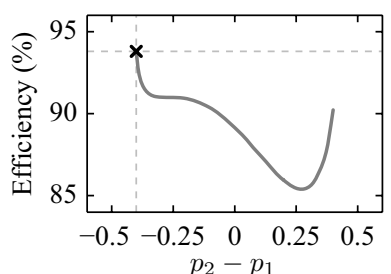

(b)

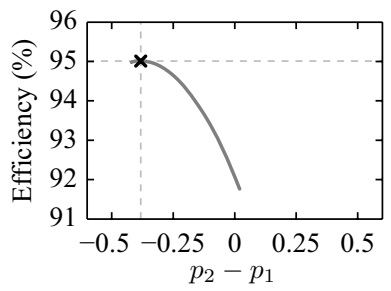

(d)
Possible system efficiency
$\times \quad$ Solution found by the proposed methodology

Figure 4: Validation of the optimization methodology for the configuration analyzed in Case 1. Optimal solution found and possible efficiency curves for (a) $0.28 \mathrm{pu}$; (b) $0.5 \mathrm{pu}$; (c) $0.57 \mathrm{pu}$ e (d) $0.78 \mathrm{pu}$.

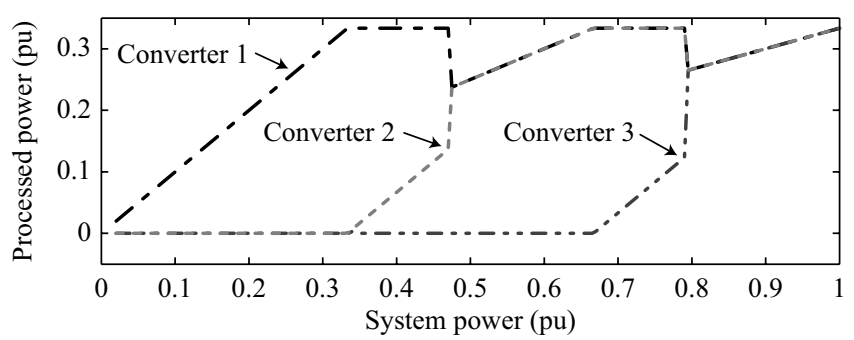

Figure 5: Power distribution curves obtained by the methodology for Case 2.

solve the multiple global minima problem. In this sense, it must be highlighted the correct operation of the Stage 4 of the methodology, since there is no unnecessary power redistributions that would result in the same maximum system efficiency, as it can be observed in Fig. 5.

\section{COnClusions}

This paper proposes a new methodology to optimize the global efficiency of systems composed by parallel converters. The nonlinearity of the problem, the constraints imposed to the solution hyperplane and the possible existence of multiple global minima makes that many numerical algorithms are not able to solve this problem properly. The proposed methodology is comprised by a global optimization stage to search for an initial guess of the point of maximum efficiency, a local optimization stage to refine results and improve precision, and an ambiguity resolution stage that is capable to chose the most appropriate global minimum in case of multiple ones. Finally, case studies are presented to validate the proposed methodology for generic cases, in which the efficiency curves and power ratings of converters are different, as well as symmetric cases, where the problem of multiple global minima occurs. The optimal solution has been properly found for all cases analyzed, making this 


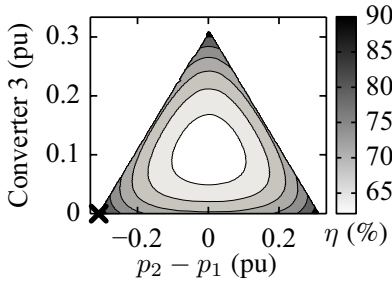

(a)

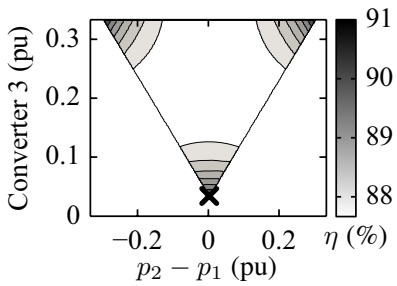

(c)

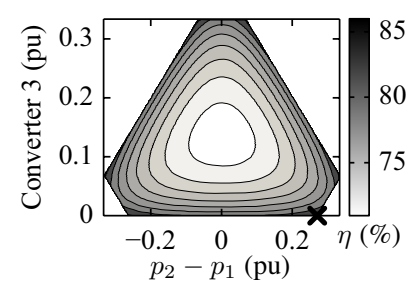

(b)

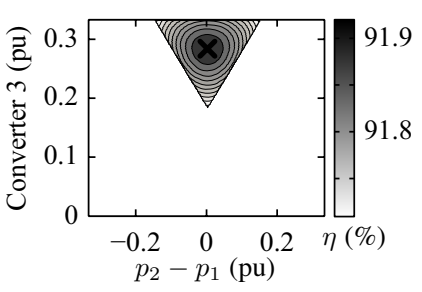

(d)
Figure 6: Validation of the optimization methodology for the configuration analyzed in Case 2. Optimal solution found and possible efficiency surfaces for (a) $0.31 \mathrm{pu}$; (b) $0.4 \mathrm{pu}$; (c) $0.70 \mathrm{pu}$ e (d) $0.85 \mathrm{pu}$.

methodology an excellent strategy for maximizing efficiency in systems of parallel converters, especially when the system works under reduced power levels for a while as in case of photovoltaic energy generation. In this sense, although with different objectives, MPPT strategies together with the proposed methodology enable the system to extract the maximum available energy and that this energy will be handled with the maximum possible efficiency, improving the economic viability of installations.

\section{ACKNOWLEDGEMENT}

This work is supported by $\mathrm{CNPq}$ (National Council of Scientific and Technological Development), CAPES (Brazilian Commission for Higher Education), FAPERGS (Foundation for Supporting Research from the State of Rio Grande do Sul), and RURALGRID project on CTP frame with support of the Comissionant per a Universitats i Recerca del Departament d'Innovació, Universitats i Empresa of the Generalitat de Catalunya.

\section{REFERENCES}

[1] F. H. Dupont, C. Rech, and J. R. Pinheiro. A methodology to obtain the equations for the calculation of the weighted average efficiency applied to photovoltaic systems. In 10th IEEE/IAS Int. Conf. on Ind. Appl., pages 1-8, 2012.

[2] J. S. Lai and D. J. Nelson. Energy management Power converters in hybrid electric and fuel cell vehicles. Proc. of the IEEE, 95(4):766-777, 2007.

[3] R. Beltrame, J. R. R. Zientarski, M. L. da Silva Martins, J. R. Pinheiro, and H. L. Hey. Simplified zero-voltagetransition circuits applied to bidirectional poles: concept and synthesis methodology. IEEE Trans. on Power Electron., $\mathrm{PP}(99): 1-1,2010$.

[4] W. Li, J. Liu, J. Wu, and X. He. Design and analysis of isolated ZVT boost converters for high-efficiency and high-step-up applications. IEEE Trans. on Power Electron., 22(6):2363-2374, 2007.
[5] Deutsche Gesellschaft für Sonnenenergie. Planning and installing photovoltaic systems: a guide for installers, architects and engineers. Earthscan, Sterling, VA, 2nd edition, 2008.

[6] A. Berasategi, C. Cabal, C. Alonso, and B. Estibals. European efficiency improvement in photovoltaic applications by means of parallel connection of power converters. In 13th Eur. Conf. on Power Electron. and Appl., pages 1-10, 2009.

[7] P. Zumel, C. Fernnndez, A. de Castro, and O. Garcia. Efficiency improvement in multiphase converter by changing dynamically the number of phases. In 37th IEEE Power Electron. Spec. Conf., pages 1-6, 2006.

[8] J. A. Abu-Qahouq, L. Huang, D. Huard, and A. Hallberg. Novel current sharing schemes for multiphase converters with digital controller implementation. In Twenty Second Annual IEEE Applied Power Electron. Conf., pages 148-156, 2007.

[9] A. Kelly. Current share in multiphase DC-DC converters using digital filtering techniques. IEEE Trans. on Power Electron., 24(1):212-220, 2009.

[10] K. Raggl, T. Nussbaumer, G. Doerig, J. Biela, and J. W. Kolar. Comprehensive design and optimization of a high-power-density single-phase boost PFC. IEEE Trans. on Ind. Electron., 56(7):2574-2587, 2009.

[11] J. Biela, U. Badstuebner, and J. W. Kolar. Design of a 5-kW, 1-U, 10-kW/dm3 resonant DC-DC converter for telecom applications. IEEE Trans. on Power Electron., 24(7):1701-1710, 2009.

[12] R. Baldick. Applied optimization: formulation and algorithms for engineering systems. Cambridge University Press, Cambridge, 2006.

[13] R. L. Haupt and D. H. Werner. Genetic algorithms in electromagnetics. IEEE Press, Hoboken, NJ, 2007.

[14] M. H. Ershadi, M. B. Poudeh, and S. Eshtehardiha. Fuzzy logic controller based genetic algorithm on the step-down converter. In Int. Conf. on Smart Manufacturing Appl., pages 324-328, 2008.

[15] M. Affenzeller, S. Winkler, S. Wagner, and A. Beham. Genetic algorithms and genetic programming: modern concepts and practical applications. CRC Press, Boca Raton, 2009.

[16] H. C. Wu. The Karush-Kuhn-Tucker optimality conditions in an optimization problem with intervalvalued objective function. European Journal of Operational Research, 176(1):46-59, 2007.

[17] S. P. Han. A globally convergent method for nonlinear programming. Journal of Optimization Theory and Applications, 22(3):297-309, 1977.

[18] M. Powell. A fast algorithm for nonlinearly constrained optimization calculations. In G. Watson, editor, Numerical Analysis, volume 630 of Lecture Notes in Mathematics, pages 144-157. Springer, Berlin, 1978.

[19] R. Fletcher. Practical methods of optimization. Wiley, Chichester, 2nd edition, 1987. 